\title{
Who defaults after treatment for gonorrhoea? Randomised controlled study of effect of an educational leaflet
}

\author{
SUSAN BEWLEY \\ From the Department of Genitourinary Medicine, James Pringle House, The Middlesex Hospital, Charlotte \\ Street, London
}

SUMMARY A study of patients with gonorrhoea showed that those who defaulted most from follow up were male, heterosexual, and had multiple sexual partners. A prospective, blind, randomised controlled trial showed that a leaflet giving information about gonorrhoea had a positive effect on follow up attendance by women, but no appreciable effect on rates of defaulting overall. This has implications for the initial treatment schedules.

It is important that patients return after treatment for gonorrhoea with antibiotics, as laboratory results are then available and it is possible to identify whether any other concurrent sexually transmitted infections have been found.' Drug resistant Neisseria gonorrhoeae, which is now found in London, ${ }^{2}$ may be present, and there may be postgonococcal urethritis. The other reason for asking patients to return is to ensure that sexual contacts have been traced. This study aimed to identify defaulters from follow up after treatment for gonorrhoea and the factors associated with nonattendance. Secondly, a health education leaflet was designed to try to improve attendance for follow up.

\section{Patients and methods}

A one month pilot study was performed in January 1985. The main study population included all patients attending this London genitourinary medicine clinic and diagnosed as having gonorrhoea between 1 April and 30 June 1985. Gonorrhoea was diagnosed if typical Gram negative intracellular diplococci were seen on microscopy or if there was a positive culture of $N$ gonorrhoeae. All patients were treated with a single dose of $3 \mathrm{~g}$ amoxycillin and $1 \mathrm{~g}$ probenicid orally witnessed by the nurses (intramuscular spectinomycin $1 \mathrm{~g}$ was used for patients who were allergic to penicillin or infected with strains known to be resistant to

Address for reprints: Dr S Bewley, Department of Obstetrics and Gynaecology, Kings College Hospital, Denmark Hill, London SE5 8RX

Accepted for publication 18 November 1987 penicillin). Patients were requested to attend one and two weeks after treatment for repeat smears and test of cure cultures.

After treatment, all patients were asked to see a health adviser on the same day, so that their sexual contacts could be traced. Patients entered the intervention trial as they left the interview with the health adviser by being handed a sealed envelope. This contained either a card with the address of the clinic, or the same card plus a leaflet about gonorrhoea and its sequelae. The leaflet was printed in bold black, red, and white on A5 paper and was divided into question and answer sections (table 1). The envelopes had been numbered and organised in randomised pairs and then into groups of 10 for women, heterosexual men, and homosexual men. This was to ensure roughly equal numbers of envelopes containing and not containing leaflets in case numbers in the three different groups of patients fluctuated.

A simple questionnaire was completed for each patient from retrospective analysis of the case notes. Patients were considered as having attended for follow up if they had been seen seven to 13 days (first follow up visit) or 14 to 21 days (second follow up visit) after treatment. To standardise follow up, visits before seven or after 21 days were not included in the study. A few visits before seven days were made by patients reattending as they were infected with penicillinase producing strains of $\mathrm{Ngonorrhoeae}$ (PPNG), and visits after 21 days were usually for unrelated conditions.

Student's $t$ test was used to test for significance. Fisher's exact test was used to deal with the small numbers in the randomised trial. A result was considered significant if $\mathrm{p}$ was $<0.05$. 
Table 1 Leaflet for patients with gonorrhoea

What is gonorrtoen?

It is an infection of the genital (sexual) organs caused by a bacterium.

What are the symptoms?

THERE MAY BE NO SYMPTOMS.

Women may notice a change in vaginal discharge, burning on passing urine, or abdominal pains.

Men may have a yellow discharge from the penis and pain on passing urine. In the rectum (back passage) it may cause discharge and soreness.

Sexual contacts of people with gonorrhoea may have caught the infection and feel entirely well.

How is it treated?

With tablets containing penicillin. If you are allergic to penicillin other antibiotics can be used. Occasionally the bacteria are resistant to penicillin and a different antibiotic is better.

Are there any complications?

Yes, but only if it is untreated.

Gonorrhoea can cause pelvic inflammatory disease in women (which causes pain and can affect fertility). It can spread to the blood (septicaemia) and joints (arthritis) and the testicles (epididymo-orchitis).

How did I catch it, and can I pass it on?

It is caught by sexual contact. It usually takes two to 10 days to get symptoms, but it may have been there longer if you had no symptoms. It is important to tell your sexual partner(s) so that they can be checked and if necessary treated. You should not start having sex again until you have been seen and given the "all-clear".

Can I have anything else?

Yes. You can catch several infections at once. Gonorrhoea is often the first to show itself and masks the others. This is another reason to come back and see us again.

Why do I need to attend for follow up if I feel well?

You may still have gonorrhoea and you need to be checked for other infections.

Does anyone have to know?

No. Everything is completely confidential.

\section{Results}

In the three months of the study 260 patients were diagnosed and treated for gonorrhoea, $236(91 \%)$ of them were interviewed by a health adviser, and only $163(61 \%)$ entered the intervention study. The 93 patients with gonorrhoea who did not enter the intervention trial consisted of 24 who did not see a health adviser because they left the clinic direct or because none was available and 73 to whom the health adviser failed to give an envelope. Four patients were withdrawn from the trial as the diagnosis of gonorrhoea was not confirmed by culture, so 159 cases were analysed. No case notes were missing.

The mean age of the 260 patients $(202$ men, 58 women) was 27 (SD 8, range 15-59). Social class was not analysed. Sexual orientation was inferred by the sex of the contacts in the preceding four weeks (although this underestimated the proportion of bisexuals). In the analysis bisexuals were arbitrarily added to the homosexual group as they constituted such a small proportion of the total population. Of the 202 men, 114 were homosexual and five bisexual, which reflects the large population of homosexual men who attend this clinic. Table 2 shows the patient characteristics.

Of the 260 patients, at least $108(42 \%)$ had previously had gonorrhoea, documented either from the history or in the case notes. This may be an underestimate as patients may attend other clinics or forget to mention previous infections. The 115 old patients had had a mean of five (range 1-26) sexually transmitted infections diagnosed at this clinic. The 56 patients who were symptomless attended because they had received a contact slip or for a check up. The diagnosis was made immediately, by microscopy, in 183 cases $(70 \%)$ and the rest were diagnosed from the culture results.

The number of different sexual partners in the preceding four weeks was used as a measure of sexual activity or the casual nature of sexual contact. The average number of sexual partners was 1.8 (range 0-14), although four, including two prostitutes, were not included as they had had "multiple" partners.

Table 3 shows that in men the most common site of gonorrhoea was the urethra, followed by the rectum, the urethra and rectum, and the pharynx. In women the infected sites, in order of incidence, were: the cervix and urethra; the cervix alone; the urethra alone; the cervix, urethra, and rectum; and the rectum alone.

Table 2 Characteristics of 260 patients diagnosed as having gonorrhoea

\begin{tabular}{lr}
\hline Characteristics & No $(\%)$ \\
\hline Men & $202(78)$ \\
Women & $58(22)$ \\
Sexuality of men: & $83(32)$ \\
Heterosexual & $114(44)$ \\
Homosexual & $5(2)$ \\
Bisexual & $193(74)$ \\
British nationality & $230(89)$ \\
London address & $145(56)$ \\
New patients & $108(42)$ \\
History of gonorrhoea & $204(79)$ \\
Symptomatic & $183(70)$ \\
Diagnosis by microscopy & $236(91)$ \\
Seen by health adviser & $163(63)$ \\
Entered into intervention trial & \\
\hline
\end{tabular}


Table 3 Site of infection with gonorrhoea in 260 patients

\begin{tabular}{lc}
\hline Site & No $(\%)$ \\
\hline Men $(\mathrm{n}=202):$ & $147(73)$ \\
Urethra & $47(23)$ \\
Rectum & $7(4)$ \\
Urethra and rectum & $1(1)$ \\
Pharynx & \\
Women (n = 58): & $35(60)$ \\
Cervix and urethra & $14(24)$ \\
Cervix & $6(10)$ \\
Urethra & $2(3)$ \\
Cervix, urethra, and rectum & $1(2)$ \\
Rectum & \\
\hline
\end{tabular}

Of the 260 patients, $186(72 \%)$ attended for the first follow up visit and $126(49 \%)$ for the second; 102 $(39 \%)$ attended one visit and $105(40 \%)$ both visits. Over all 53 patients $(20 \%)$ did not return. A closer examination was then made of this group of defaulters-patients who did not attend either follow up visit.

The proportion of the 260 patients who defaulted varied according to their sex and sexuality; five of the 58 women, 24 of the 119 homosexual men, and 24 of the 83 heterosexual men defaulted $(p<0.05)$. Men with urethral gonorrhoea might be more easily able to tell that the infection has been successfully treated than men with rectal gonorrhoea or women and therefore default more from follow up. To see whether this difference was a function of sexuality or of different presentations of the disease, defaulting was compared by site of infection. The difference in proportions of defaulters between homosexual and heterosexual men $(20 / 107 v 24 / 83)$ remained even when those with urethral gonorrhoea alone were compared $(11 / 60 v 24 / 83)$. The proportion of homosexual men who defaulted was similar whether they had urethral $(11 / 60)$ or rectal $(9 / 47)$ gonorrhoea. This evidence suggests that, for men, sexual orientation, rather than site of infection, affected attendance at follow up.

The other major important factor affecting follow up was the number of sexual partners in the previous four weeks; $43 \%$ (16/37) of patients with three or more sexual partners failed to attend for follow up compared with $17 \%(37 / 223)$ with two or fewer $(\mathrm{p}=0.003)$.

Factors not associated with failure to attend for follow up were age, nationality, address, presence or absence of symptoms, whether the diagnosis was made by microscopy or culture, the health adviser or doctor seen, site of infection, history of gonorrhoea, and the number of previous diagnoses at this clinic.

\section{INTER VENTION STUDY}

The same proportion of patients given a leaflet (17/82) as of those not given a leaflet (15/77) defaulted. The leaflet was related, however, to a better follow up attendance of women; all of 18 women given a leaflet returned for follow up, whereas four of the 17 women not given a leaflet defaulted completely (Fisher's exact test $=0.045$ ). Even though the two groups were randomised, they were checked to see that there was no difference in patient characteristics to account for this finding. The only difference found was that the two prostitutes with multiple partners were in the group not given a leaflet. The difference in defaulting rates remained appreciable even when the two prostitutes with multiple partners were excluded.

For the main study, to detect a difference in defaulting from $20 \%$ to $15 \%$ with a power of $0.05,186$ subjects would have been needed in each arm of the trial. The numbers of patients with gonorrhoea was smaller than expected from the pilot study, and because of illness and forgetfulness, too few patients were recruited during the study period. Despite these limitations a difference was seen for women.

\section{Discussion}

In this retrospective study the main associations with defaulting from follow up were found to be male gender, heterosexuality if a man, and having more than three sexual partners in the preceding month. The intervention, giving an information leaflet about gonorrhoea, had no appreciable effect on the rates of follow up over all, but seemed to improve the default rate in women. Although they already attend for follow up more assiduously than men, they are also most at risk of serious complications from gonorrhoea. They may have asymptomatic disease or concurrent chlamydial infection, and can develop salpingitis, pelvic inflammatory disease, chronic pelvic pain, or infertility. ${ }^{3-5}$ The total number of cases of gonorrhoea in Britain in 1985 was $53802,{ }^{6}$ although the incidence may be falling, at least in homosexual men. ${ }^{7}$

This study confirms similar results of other investigators. Of 147 men with urethral gonorrhoea attending this clinic only $103(70 \%)$ returned at one week and $88(60 \%)$ at two weeks (Tovey and Carne, personal communication). Perferement and Overfield studied 100 patients with all diagnoses of STDs, who were interviewed prospectively, and 32 defaulted. ${ }^{8}$ Patients were more likely to default if they had been to other clinics, if they were of "lower professional" social class, if they were women with candidiasis, and if their perception of sexually transmitted diseases had changed during their visit from "embarrassing" to "ordinary". They were less likely to default if their attitude was positive before attending the clinic. No relation was found with age or gender. Our study only looked retrospectively at patients with gonorrhoea and was unable to assess their attitude, but did find a strong relation with sex, sexuality, and sexual activity. 
To see whether counselling affected follow up, Goodrich studied 100 consecutive patients with gonorrhoea and gave counselling about gonorrhoea to half (educated group) and general counselling to the other half (uneducated group). ${ }^{9}$ The default rates were $10 \%$ in the educated and $25 \%$ in the uneducated groups. Though this difference was appreciable, there may have been more patients with symptomatic postgonococcal urethritis (PGU) in the first group. It was also felt that an average of 25 minutes counselling per patient was an expensive use of resources.

Patients should attend for follow up even if their symptoms have abated. The estimate of the proportion of asymptomatic disease varies, but may be as high as $25 \%{ }^{10}$ to $60 \%$. $^{5}$ These people certainly need a test of cure as symptomless men and women act as resevoirs of infection."

Many patients with gonorrhoea have other associated infections, especially PGU and chlamydial infection, and should return for culture results and repeat testing. Chlamydia trachomatis has been found in $20 \%$ of women and $30 \%$ of men with gonorrhoea. ${ }^{\prime}$ A return visit gives the doctors an opportunity to check on the success of contact tracing.

The implications of this study are twofold. Firstly, if the probable defaulters are identifiable then efforts can be directed to encourage them to attend. Secondly, it may be that a hard core of patients, despite all effort, will never reattend the clinic, and treatment schedules should reflect this reality. Extra health education interventions, such as seeing a health adviser, being counselled, or receiving an information leaflet may have a small effect on default rates. Written material is thought to help patients to recall information, although large studies are required to prove any beneficial effect. ${ }^{12}$ This study suggests that the largest effect will be seen in the most motivated groups. If there are patients who are resistant to reattending, management might have to be altered. Access to a clinic to provide repeat smears should be easy. All patients should see a contact tracer on their first visit, preferably before completion of treatment, to increase compliance and possibly influence behaviour. Although genitourinary doctors disapprove of epidemiological treatment of infections, the results of this study would support simultaneous prophylactic treatment for PGU in promiscuous men with gonorr- hoea, especially if they are heterosexual, as untreated undiagnosed chlamydial infection can be both symptomless and damaging in the female reproductive organs. ${ }^{3414}$ As infections can be both symptomless and potentially dangerous in women, and they do reattend the clinic this study would not lend support to reducing their follow up.

I thank Mr P Williams for statistical advice, Professor M W Adler for his support, and the clinic staff at James Pringle House for their help in conducting this study.

\section{References}

1 Oriel J. Chlamydia trachomatis and postgonoccocal genital infection. In: Hobson D, Holmes KK, eds. Nongonococcal urethritis and related infections. Washington DC: American Society for Microbiology, 1977:230-2.

2 Thin RN, Barlow D, Eykyn S, Phillips I. Imported penicillinase producing Neisseria gonorrhoeae becomes endemic in London. British Journal of Venereal Diseases 1983;59:364-8.

3 Weström L. Effect of acute pelvic inflammatory disease on fertility. Am J Obstet Gynecol 1975;121:707-13.

4 Weström L. Incidence, prevalence and trends of acute pelvic inflammatory disease and its consequences in industrialized countries. Am J Obstet Gynecol 1980;138:880-92.

5 Catterall RD. A short textbook of venereology: the sexually transmitted diseases. Sevenoaks: Hodder and Stoughton, 1974:14-37.

6 Department of Health and Social Security. Sexually transmitted diseases. In: On the state of the public health for the year 1984. (The annual report of the chief medical officer of the Department of Health and Social Security for the year 1984.) London: HMSO, 1986:49-54.

7 Weller I, Hindley D, Adler M, Meldrum J. Gonorrhoea in homosexual men and media coverage of the acquired immune deficiency syndrome in London 1982-3. Br Med J 1984; 289:1041.

8 Perferement $\mathrm{S}$, Overfield $\mathrm{K}$. Which patients with sexually transmitted diseases default? British Journal of Venereal Diseases 1978;54:201-4.

9 Goodrich KK. Gonococcal infection: the effect of educational counselling on patient compliance. British Journal of Venereal Diseases 1981;57:137-40.

10 Brooks G, Donegan E. Gonococcal infections in women. In: Gonococcal infections. London: Edward Arnold, 1985:85-104.

11 Brooks G, Donegan E. Salpingitis. In: Gonococcal infections. London: Edward Arnold, 1985:105-20.

12 Barnes RC, Holmes KK. Epidemiology of gonorrhea: current perspectives. Epidemiol Rev 1984;6:1-30.

13 Drury VWM. Patient information leaflets. $\mathrm{Br}$ Med $\mathrm{J}$ 1984;288:427-8.

14 Curran JW. Economic consequences of pelvic inflammatory disease in the United States. Am J Obstet Gynecol 1980;138: 848-51. 